\title{
Needed relapse-prevention research on novel framework (ASPIRE model) for substance use disorders treatment
}

\author{
Udi E. Ghitza* \\ Center for the Clinical Trials Network, National Institute on Drug Abuse, National Institutes of Health, Bethesda, MD, USA \\ *Correspondence: ghitzau@nida.nih.gov \\ Edited by: \\ Marco Diana, University of Sassari, Italy \\ Reviewed by: \\ Angelo Giovanni Icro Maremmani, University of Pisa, Italy \\ Marco Diana, University of Sassari, Italy
}

Keywords: addictive disorders, drug abuse, substance abuse, addiction, addiction treatment, drug abuse treatment, personalized medicine, precision medicine

\section{INTRODUCTION}

According to a recent United States (U.S.) national survey on drug use and health, substance use disorders (SUDs) characterized by hazardous use of alcohol, nonprescribed drugs, and illicit drugs are common. In 2013, 22.7 million persons ( $8.6 \%$ of persons aged 12 years or older) needed treatment for an illicit drug or alcohol-use problem (1). Drug use disorders produce a wide variety of medical problems and are important contributors to years of life lost due to disability and preventable death (2). Yet, screening, intervening, and treating SUDs have not been embraced within general medical settings (3). In fact, even though untreated SUDs place individuals at substantially greater risk for a wide range of diseases and are a pervasive public health burden, only approximately one-tenth of Americans aged 12 years or older with SUDs received treatment in 2013 (1). A main obstacle to patient engagement in and initiation of SUDs treatment is lack of motivation to seek out follow-up care. Motivation to do so may be compromised by a lack of a personalized-medicine approach, in which patients are frequently only offered a single or narrow set of care options, which do not align with their individual needs. A gap exists in research in medical settings regarding how to effectively implement shared-decision making between patients and their providers, concerning how to tailor evidence-based care options to personalized needs and preferences of clients (4-6).

Converging research over the past 30 years indicates that core features of
SUDs comprise of a spectrum of neurobiological components, which in 2014, I distilled into a shared-decision-making and personalized/precision medicine framework for SUD treatment, the ASPIRE model (7). This integrative model stresses the need for translational research on how to most effectively optimize implementation of patient-centered care using this model in general medical settings and specialty SUD-treatment programs, in a manner whereby patients have an active role as partners in deciding with their providers on treatment strategies for evidence-based care tailored to their risk categories and values (7). This precision medicine approach should be customized to genetic, psychological, and physiological profiles and environmental events that patients experience as presenting an obstacle to their overall wellness and recovery (7). Accordingly, treatment and follow-up care plans should be matched with individual disease characteristics, which may enhance motivation of patients to cease hazardous substance use and associated health risk behaviors (7). Thus, the ASPIRE model uses as its foundational principle shared-decision-making between patients and healthcare providers to customize personalized medical care to particular neuroscience-research-grounded profiles, psychiatric comorbidities, readiness to change, risk categories, and problems that individual patients report as most distressing to their daily lives (7). Recent advances in genomic and health information technologies [for instance, meaningful use of electronic health record systems (EHRs), telemedicine, mobile-health technologies, EHRs-linked patient registries, EHRslinked genomic data and lifestyle information, etc.] and bioinformatics methods for analyzing EHRs-linked biomedical bigdata repositories should be leveraged to accelerate rigorous big-data science research on how to effectively implement such a personalized-medicine framework in general medical settings.

In circumstances where individual patients are cognitively impaired and have limited insight, such a shared-decisionmaking approach to SUD treatment may actively involve patients' caregivers or other family members, if patients consent to their involvement in their medical care (47). In such cases, family members may be recruited to help ensure patients follow through and adhere to their treatment plan mutually agreed upon with their medical provider. Systematic rigorous research is needed using a shared-decision-making approach to find more effective ways of reducing the burden of SUD and enhancing quality of lives not only for individual patients but also for their family members affected by an individual's SUD.

\section{EVIDENCE GAPS AND RESEARCH AREAS CONCERNING ASPIRE FRAMEWORK FOR PATIENT-CENTERED TREATMENT OF SUDS}

For patients at the severe end of the SUDs spectrum, below I illuminate needed relapse-prevention research testing combined evidence-based cognitive-behavioral therapy and pharmacological-intervention adjuncts targeting prominent components in the ASPIRE framework. " $A$ " in this 
framework denotes anhedonia/rewarddeficit and " $S$ " a stressful state, referring to a sensitized brain anhedonia and stress system following repeated heavy drug use and during drug withdrawal (8). This sensitized brain system produces deficits in responses of the brain to rewarding stimuli and produces a negative emotional state, which drives continued drug-taking behavior (8). " $P$ " denotes a pathological lack of self-control to cut down drug use despite negative implications, which involves impairments in prefrontal-cortical cognitive regulation of inhibitory behaviors to reduce drug taking. Relevant to these phenotypes, recent converging clinical-research findings suggest an overlap among neurobiological events mediating drug craving and relapse triggered by stress and drug-predictive environmental cues (9-13). Across various SUDs (e.g., cocaine, opioid, and tobacco use disorders), these findings indicate adrenergic alpha-2 receptor agonists (e.g., guanfacine, clonidine, lofexidine) may have promise for facilitating relapse prevention by decoupling brain responses to stress and drug-predictive cues from drug craving (9-12). Further, guanfacine is approved by the U.S. Food and Drug Administration (FDA) for treating attention deficit hyperactivity disorder (ADHD) in youth. It has been shown to enhance cognitive processes involved in response inhibition and executive functioning, such as attention shifting, thereby facilitating cortical regulatory control over inflexible behaviors associated with vulnerability to drug seeking and taking in individuals with SUDs $(11,14)$.

Based upon the above promising proofof-concept clinical studies, double-blind placebo-controlled randomized clinical trials (RCTs) are needed in treatmentseeking populations to test whether guanfacine or other adrenergic alpha-2 receptor agonists may be effective as pharmacotherapy adjuncts to augment efficacy of currently available evidence-based treatment options, to enhance relapse prevention in recently abstinent patients.

For instance, in the case of opiateuse disorders, there are effective FDAapproved medication-assisted treatment (MAT) options (such as buprenorphine in medical settings). However, even with these treatment options, opiate-use relapse rates remain high. Thus, comparative effectiveness research is needed in general medical settings to determine whether adrenergic alpha-2 receptor agonist adjuncts thought to work by different mechanisms of action to reduce opiate use-enhance the effectiveness of buprenorphine in relapse prevention, compared with buprenorphine alone.

It would be helpful for such RCTs to follow the FDA guidance on enrichment strategies for clinical trials to support approval of human drugs (15). For example, patients at study intake could be queried on whether they experience frequent psychological distress from stress or restlessness/impulsivity. This is because adrenergic alpha-2 receptor agonists are postulated to be beneficial in relapse prevention by decoupling sensitized brain responses to stress from drug craving, reducing impulsivity, and enhancing cognitive processes important for attention shifting (11-14). If patients do report experiencing such psychological symptoms, the likelihood of detecting an efficacy signal may be improved by employing a personalized-medicine approach in which these patient subgroups would be included in these relapse-prevention trials, since they are likely to benefit most from these agents.

Furthermore, in the context of precision medicine, adaptive designs research is needed to develop and test outpatient treatment algorithms and actionable clinical decision support for different patient subgroups with opiate-use disorders, with or without chronic pain, which incorporate shared-decision making practices in improving their health outcomes and reducing the risk of death from overdose and other opiate-use related negative consequences.

" $I$ " and " $R$ " in the ASPIRE model denote insomnia and restlessness, common SUDs withdrawal symptoms following repeated heavy drug use that patients find distressing, increasing likelihood of relapse following abstinence (16-20). Gabapentin is a widely prescribed FDA-approved anticonvulsant medication, which reduces alpha2d-1 membrane trafficking and calcium currents at voltage-gated calcium channels in regions involved in the brain's sensitized responses to stress following repeated heavy drug use and withdrawal $(21,22)$. Gabapentin has also been frequently used off-label to reduce sleep disturbances and restlessness. Recent clinical research suggests gabapentin may have promise in reducing alcohol and cannabis use and craving in alcohol- and cannabisdependent individuals, as well as sleep and mood disturbances $(21,22)$. Randomized controlled trials are needed to test gabapentin's effectiveness in facilitating relapse prevention in recently abstinent cannabis-use and/or alcohol-use disorder treatment seekers, employing the FDA guidance on enrichment strategies for clinical trials to support approval of human drugs (15). If patients report experiencing sleep disturbances or restlessness impairing their daily lives, they would be considered for such a RCT, which would test effectiveness of gabapentin combined with suitable evidence-based cognitivebehavioral therapies to target these disturbances, enhance duration of abstinence, and prevent relapse.

In the ASPIRE model, " $E$ " denotes excessive preoccupation with seeking drug reinforcement, compared with natural reinforcers, after transition from volitional to compulsive drug use, especially following drug craving when an individual with a SUD comes in contact with a drugassociated environment. Convergent preclinical research, using the reinstatement model of drug relapse, suggests glutamate signaling dysfunction in the mesocorticolimbic dopamine system plays a key role in this addictive process across multiple substances of abuse, including alcohol, cannabis, cocaine, nicotine, and opiates (23). N-acetylcysteine is an $\mathrm{N}$ acetyl pro-drug of the naturally occurring amino acid cysteine and is FDA-approved as a mucolytic agent for bronchopulmonary disorders (24). Preclinical research indicates that $\mathrm{N}$-acetylcysteine works to reduce drug-environment-triggered reinstatement of drug seeking for various drugs of abuse (23). It may do so by normalizing imbalances in glutamate signaling and associated pathophysiology in neuroplasticity in the mesocorticolimbic dopamine system, in part by reversing downregulation of glial-glutamate transporter1 (GLT-1) and metabotropic glutamate receptor $2 / 3$ (mGluR2/3) function $(25,26)$. 
The National Drug Abuse Treatment Clinical Trials Network of the National Institute on Drug Abuse (NIDA CTN) is currently implementing a nationwide placebo-controlled RCT evaluating efficacy of $\mathrm{N}$-acetylcysteine versus placebo, added to contingency management, for cannabis cessation in adults ages $18-50$ years old. The basis for this trial is positive findings from a RCT in cannabis-dependent adolescents, which found more than twice greater odds of cannabis abstinence during treatment with $\mathrm{N}$-acetylcysteine compared with placebo $(27,28)$. Since basic research suggests that the mechanism of action by which $N$-acetylcysteine works may suit it as a relapse-prevention agent, RCTs are also needed to test its efficacy as a relapse-prevention adjunct to evidence-based treatment options in recently abstinent treatment seekers. Further, pharmacological adjuncts need to be tested, which operate by similar mechanisms to reverse brain-reward-system down-regulation of GLT- 1 and mGluR2/3 function and associated glutamate signaling imbalances, but which have greater potency, bioavailability, and membrane permeability than $\mathrm{N}$-acetylcysteine. For instance, $N$-acetylcysteine amide (NACA) is a more potent derivative with greater bioavailability and membrane permeability, which may translate into improved clinical research use as a relapse-prevention candidate $(23,29)$.

\section{CONCLUSION}

Relapse-prevention research is needed in the above areas using a personalized/precision medicine approach in which medical decisions regarding treatment options are tailored to patient subgroups' disease profiles and risk categories. The ASPIRE model for SUDs treatment takes into account needs for instituting a shareddecision approach over the course of patient-centered care, addressing preferences and values of patients and their families (7). This framework calls for rigorous systematic research on effectiveness of personalizing treatment to those phenotypes that patients report as presenting distressing problems to their daily lives, driving their continued drug-taking behaviors, and increasing likelihood of drug relapse (7). The FDA guidance on enrichment strategies for clinical trials to support approval of human drugs (15) should be used to guide the design of randomized controlled trials in a manner matching interventions and their mechanisms of action to personalized needs of patients. It would be useful for enrichment strategies to be implemented in a manner whereby only subgroups most likely to benefit from interventions' postulated mechanism-of-action are included at study intake, prior to randomization. The likelihood of detecting an efficacy signal may be improved following such an approach. Clinical research programs, such as NIDA's National Drug Abuse Treatment Clinical Trials Network (CTN), can serve an important role in furthering such studies. Existing health information system infrastructures in practice-based research networks and recent advances in bioinformatics methods for analyzing biomedical big-data repositories of large patient cohorts should be leveraged to help accelerate this line of personalizedmedicine research. This, in turn, could lead to more cost-efficient enrollment of patients into trials, increased recruitment of providers to deliver interventions within studies, and more efficient utilization of EHRs, EHRs-linked genetic information, mobile-health and telemedicine technologies, and EHRs-linked patient registries $(3,7,30)$.

\section{ACKNOWLEDGMENTS}

UG is an employee of the Center for the Clinical Trials Network, NIDA, which is the funding agency for the National Drug Abuse Treatment Clinical Trials Network. The opinions in this paper are those of the author and do not represent the official position of the U.S. government.

\section{REFERENCES}

1. Substance Abuse and Mental Health Services Administration. Results from the 2013 National Survey on Drug Use and Health: Summary of National Findings. Rockville, MD: Substance Abuse and Mental Health Services Administration (2014).

2. Degenhardt L, Whiteford HA, Ferrari AJ, Baxter AJ, Charlson FJ, Hall WD, et al. Global burden of disease attributable to illicit drug use and dependence: findings from the global burden of disease study 2010. Lancet (2013) 382:1564-74. doi:10.1016/S0140-6736(13)61530-5

3. Ghitza UE, Tai B. Challenges and opportunities for integrating preventive substance-use-care services in primary care through the Affordable Care Act. J Health Care Poor Underserved (2014) 25:36-45. doi:10.1353/hpu.2014.0067
4. Bradley KA, Kivlahan DR. Bringing patientcentered care to patients with alcohol use disorders. JAMA (2014) 311:1861-2. doi:10.1001/jama. 2014.3629

5. Légaré F, Witteman HO. Shared decision making: examining key elements and barriers to adoption into routine clinical practice. Health Aff (Millwood) (2013) 32:276-84. doi:10.1377/hlthaff. 2012.1078

6. Elwyn G, Frosch D, Thomson R, Joseph-Williams N, Lloyd A, Kinnersley P, et al. Shared decision making: a model for clinical practice. J Gen Intern Med (2012) 27:1361-7. doi:10.1007/s11606-0122077-6

7. Ghitza UE. ASPIRE model for treating cannabis and other substance use disorders: a novel personalized-medicine framework. Front Psychiatry (2014) 5:180. doi:10.3389/fpsyt.2014.00180

8. Wise RA, Koob GF. The development and maintenance of drug addiction. Neuropsychopharmacology (2014) 39:254-62. doi:10.1038/npp.2013.261

9. Jobes ML, Ghitza UE, Epstein DH, Phillips KA, Heishman SJ, Preston KL. Clonidine blocks stressinduced craving in cocaine users. Psychopharmacology (Berl) (2011) 218:83-8. doi:10.1007/ s00213-011-2230-7

10. McKee SA, Potenza MN, Kober H, Sofuoglu M, Arnsten AF, Picciotto MR, et al. A translational investigation targeting stress-reactivity and prefrontal cognitive control with guanfacine for smoking cessation. J Psychopharmacol (2014). doi: 10.1177/0269881114562091

11. Fox H, Sinha R. The role of guanfacine as a therapeutic agent to address stress-related pathophysiology in cocaine-dependent individuals. Adv Pharmacol (2014) 69:217-65. doi:10.1016/ B978-0-12-420118-7.00006-8

12. Sinha R, Kimmerling A, Doebrick C, Kosten TR. Effects of lofexidine on stress-induced and cueinduced opioid craving and opioid abstinence rates: preliminary findings. Psychopharmacology (Berl) (2007) 190:569-74. doi:10.1007/s00213006-0640-8

13. Fox HC, Tuit KL, Sinha R. Stress system changes associated with marijuana dependence may increase craving for alcohol and nicotine. Hum Psychopharmacol (2013) 1:40-53. doi:10. 1002/hup. 2280

14. Fox H, Sofuoglu M, Sinha R. Guanfacine enhances inhibitory control and attentional shifting in early abstinent cocaine-dependent individuals. J Psychopharmacol (2015). doi:10.1177/ 0269881114562464

15. U.S. Food and Drug Administration (FDA). Guidance for Industry: Enrichment Strategies for Clinical Trials to Support Approval of Human Drugs and Biological Products. Silver Spring, MD: FDA (2012).

16. Babson KA, Boden MT, Bonn-Miller MO. The impact of perceived sleep quality and sleep efficiency/duration on cannabis use during a self-guided quit attempt. Addict Behav (2013) 38:2707-13. doi:10.1016/j.addbeh.2013.06.012

17. Allsop DJ, Norberg MM, Copeland J, Fu S, Budney AJ. The cannabis withdrawal scale development: patterns and predictors of cannabis withdrawal and distress. Drug Alcohol Depend (2011) 119:123-9. doi:10.1016/j.drugalcdep.2011. 06.003 
18. Brooks AT, Wallen GR. Sleep disturbances in individuals with alcohol-related disorders: a review of cognitive-behavioral therapy for insomnia (CBT-I) and associated non-pharmacological therapies. Subst Abuse (2014) 8:55-62. doi:10.4137/ SART.S18446

19. Matuskey D, Pittman B, Forselius E, Malison RT, Morgan PT. A multistudy analysis of the effect of early cocaine abstinence on sleep. Drug Alcohol Depend (2011) 115:62-6. doi:10.1016/j. drugalcdep.2010.10.015

20. Hughes JR. Effects of abstinence from tobacco: valid symptoms and time course. Nicotine Tob Res (2007) 9:315-27. doi:10.1080/14622200701188919

21. Mason BJ, Quello S, Goodell V, Shadan F, Kyle M, Begovic A. Gabapentin treatment for alcohol dependence: a randomized controlled trial. JAMA Intern Med (2014) 174:70-7. doi:10.1001/ jamainternmed.2014.1591

22. Mason BJ, Crean R, Goodell V, Light JM, Quello $\mathrm{S}$, Shadan $\mathrm{F}$, et al. A proof-of-concept randomized controlled study of gabapentin: effects on cannabis use, withdrawal and executive function deficits in cannabis-dependent adults. Neuropsychopharmacology (2012) 37:1689-98. doi:10.1038/npp.2012. 14

23. McClure EA, Gipson CD, Malcolm RJ, Kalivas PW, Gray KM. Potential role of $\mathrm{N}$-acetylcysteine in the management of substance use disorders. CNS Drugs (2014) 28:95-106. doi:10.1007/s40263-0140142-x

24. Grandjean EM, Berthet P, Ruffman R, Leuenberger P. Efficacy of oral long-term $\mathrm{N}$-acetylcysteine in chronic bronchopulmonary disease: a metaanalysis of published double-blind, placebocontrolled clinical trials. Clin Ther (2000) 22:209-21. doi:10.1016/S0149-2918(00)88479-9

25. Reissner KJ, Gipson CD, Tran PK, Knackstedt LA, Scofield MD, Kalivas PW. Glutamate transporter GLT-1 mediates N-acetylcysteine inhibition of cocaine reinstatement. Addict Biol (2014) 20(2):316-23. doi:10.1111/adb.12127

26. Reissner KJ, Brown RM, Spencer S, Tran PK, Thomas CA, Kalivas PW. Chronic administration of the methylxanthine propentofylline impairs reinstatement to cocaine by a GLT-1dependent mechanism. Neuropsychopharmacology (2014) 39:499-506. doi:10.1038/npp.2013.223

27. Gray KM, Carpenter MJ, Baker NL, DeSantis SM, Kryway E, Hartwell KJ, et al. A double-blind randomized controlled trial of $\mathrm{N}$-acetylcysteine in cannabis-dependent adolescents. Am J Psychiatry (2012) 169:805-12. doi:10.1176/appi.ajp.2012. 12010055

28. McClure EA, Sonne SC, Winhusen T, Carroll KM, Ghitza UE, McRae-Clark AL, et al. Achieving cannabis cessation - evaluating $\mathrm{N}$-acetylcysteine treatment (ACCENT): design and implementation of a multi-site, randomized controlled study in the national institute on drug abuse clinical trials network. Contemp Clin Trials (2014) 39:211-23. doi:10.1016/j.cct.2014.08.011

29. Sunitha K, Hemshekhar M, Thushara RM, Santhosh MS, Yariswamy M, Kemparaju K, et al. $N$-acetylcysteine amide: a derivative to fulfill the promises of $\mathrm{N}$-acetylcysteine. Free Radic Res
(2013) 47:357-67. doi:10.3109/10715762.2013. 781595

30. Tai B, Sparenborg S, Ghitza UE, Liu D. Expanding the national drug abuse treatment clinical trials network to address the management of substance use disorders in general medical settings. Subst Abuse Rehabil (2014) 5:75-80. doi:10.2147/ SAR.S66538

Conflict of Interest Statement: The author declares that the research was conducted in the absence of any commercial or financial relationships that could be construed as a potential conflict of interest.

Received: 20 January 2015; paper pending published: 12 February 2015; accepted: 21 February 2015; published online: 06 March 2015.

Citation: Ghitza UE (2015) Needed relapse-prevention research on novel framework (ASPIRE model) for substance use disorders treatment. Front. Psychiatry 6:37. doi: 10.3389/fpsyt.2015.00037

This article was submitted to Addictive Disorders and Behavioral Dyscontrol, a section of the journal Frontiers in Psychiatry.

Copyright (c) 2015 Ghitza. This is an open-access article distributed under the terms of the Creative Commons Attribution License (CC BY). The use, distribution or reproduction in other forums is permitted, provided the original author(s) or licensor are credited and that the original publication in this journal is cited, in accordance with accepted academic practice. No use, distribution or reproduction is permitted which does not comply with these terms. 